\title{
Cateterismo venoso central por vía percutánea
}

\author{
Dr. Enrique Paris M. ${ }^{1}$; Dr. Jaime Cordero Th. ${ }^{1}$
}

\section{Percutaneous catheterization of superior vena cava through the internal yugular vein}

A prospective study was held between January 1986 and April 1987 to evaluate the use of percutaneous central catheters through internal jugular vein; 47 childrens were catheterized during a 15 months period. Right jugular vein was the most frequent site of puncture. The age range of patients varied from 2.5 months and 14 years old; their varied between $2.5 \mathrm{~kg}$ and $37 \mathrm{~kg}$. The catheters were in use for an average of 4.2 days, range 1 to 17 days. Complications consisted in carotjd arterial punction in 4 patients, pneumothorax in 2 cases and transitory bradichardia in I case.

(Key words: catheterization, central venous, indwelling catherers, internal jugular ven.)

El cateterismo de las grandes venas es importante en la monitoría de los pacientes críticos y en la alimentación parenteral a largo plazo ${ }^{1,2}$. En una emergencia con hipovolemia es útil conocer la técnica que permite un acceso rápido a una vía central y el aporte consecuente de volumen ${ }^{3}$.

Se realizó un trabajo prospectivo que abarcó el período enero 86-abril 87 y cuya finalidad fue evaluar la efectividad de la técnica, el motivo de uso, el tiempo de uso y las complicaciones de la cateterización de la vena cava superior por vía de la punción percutánea de la vena yugular interna.

\section{MATERIAL Y METODO}

Se practicó el cateterismo de la vena cava superior en 49 niños. Se intentó colocar 51 catéteres, 2 niños fueron cateterizados en 2 oportunidades, empleando la técnica de abordaje alto y central, citado por Prince y colaboradores ${ }^{4}$. Esta técnica consiste en ura punción de la vena yugutar interna, tenjendo como base anatómica el triángulo que forma el músculo esternocleido mastoideo al dividirse en dos ramas, una de las cuales se inserta en el esternón; y la otra, en el extremo medial de la clavícula respectiva. La base del triángulo está entre la clavícula y el esternón, y el ápice hacia la extremidad cefálica a una altura variable según el tamaño del paciente. Una vez ubicado este triángulo se punciona en el ángulo superior dirigiendo la aguja de punción hacia el tórax, en dirección a la inamila homolateral y levantando la jeringa, en la que va montada la aguja, que va llena de suero fistológico con un baño de hepa-

1. Unidad de Cuidados Intensivos, Hospital Luis Calvo Mackenna. rina, en un ángulo de $30^{\circ}$ y aspirando, en forma continua. Cuando se obtiene reflujo de sangre venosa se detiene el avance, se retira la jeringa y por el lumen de la aguja se introduce la guía de alanbre y luego, por fuera de ésta, el catéter elegido.

Se uttlizaron 2 tipos de catéteres; catéteres tipo Seldinger (Certofix 18 de Braun (R) o Leadercat 18 de Yygon (R), y catéteres típo intracatéter (Cavafix 18 de Braun (R). Los niños que iban a ser cateterizados fueron sedados con morfina IM a dosis de 0,08 a 0,1 $\mathrm{mg} / \mathrm{kg}$, se inmovilizaban, se colocaban en posición de Trendelenburg y con la extremidad cefálica rotada hacia el lado opuesto de la vena elegida.

Una vez colocado el catéter éste fue controlado siempre con una radiografía de tórax AP portátil para asegurar una buena posición (extremidad distal del catéter en la entrada de la cava a la aurícula derecha); posteriormente se fijó con tela adhesiva previa cubierta con gasa enbebida en povidona yodada. Al tetirar el catéter se cultivó el extremo distal de éste o se tomó un hemocultivo previo a su retiro, por vía del mismo catéter.

Las catéteres oolocados por la técnica de Seldinger fueron reemplazados cada 7 días aproximadamente; esta técnica permite un fácil reemplazo del catéter ya que no es necesario volver a puncionar, haciéndose e] cambio sólo utilizando la guía de alambre ${ }^{\mathrm{s}}$.

\section{RESULTADOS}

Se hicieron 51 intentos para colocar catéteres centrales por vía yugular en el periodo de estudio, con buen éxito en 47 casos. Las edades de los pacientes fluctuaron entre los 2,5 meses y los 14 años, incluyendo 16 pacientes menores de 6 meses y 9 mayores de 5 años. El peso de los nifros de la serie varió entre 2,5 y $37 \mathrm{~kg}, 11 \mathrm{pa}$ cientes pesaban menos de $5 \mathrm{~kg}$ y 24 más de $10 \mathrm{~kg}$. 
La vía de abordaje fue habitualmente la yugular interna derecha que, anatómicamente, está en la misma dirección de la cava superior en su trayecto hacia la aurícula derecha; ocasionalmente se intentó cateterizar por vía de la yugular interna izquierda (en casos de lesiones a derecha o de punciones previas) que confluye hacia la cava superior por la innominada y sigue, por lo tanto, un trayecto sinuoso. Se logró instalar 41 catéteres por vía de la yugular interna derecha y 6 catéteres por la yugular interna izquierda.

En lo referente a las indicaciones para la instalación de una via venosa central, ésta fue utilizada para aportar liquidos (sueros, derivados sanguíneos 0 albúmina humana) en 47 niffos, nutricion parenteral total (NPT) en 13 pacientes y medir presión venosa central (PVC) en 29 casos. En algunos pacientes la vía se utilizó para más de un fin.

E1 catéter tipo Seldinger se empleó en 39 oportunidades y el intracatéter en 8.

El tiempo de permanencia del catéter fue, en promedio, de 4,2 dias, con un rango que fue desde 1 día hasta 17 dias de uso. En 35 pacientes el catéter fue utilizado durante 7 dias o menos. En 12 pacientes se usó durante más de 7 días, alcanzando el tiempo más prolongado un caso con 17 días de uso.

Tuvimos en nuestra serie tres tipos de complicaciones que fueron: punción de la arteria caró tida en 4 oportunidades, neumotórax en 2 oportunidades y bradicardia transitoria en un caso. Esto arroja porcentajes de $7,8 \%, 3,9 \%$ y $1,85 \%$, respectivamente.

Como fue expresado en Material y Métodos, al ser retirados los catéteres se cultivaron o se tomó un hemocultivo a través del catèter en el momento de extraerlo. Los gérmenes encontrados fueron: Staphylococcus albus en tres casos, Candida en 1 caso y Micrococcus $s p$ en 1 caso. En ninguno de los pacientes en los cuales se encontraron los gérmenes citados se volvió a hallar el mismo germen en un hemocultivo separado, tomado a distancia $y$ en forma concomitante.

\section{DISCUSION}

La cateterización de una vena central por vía percutánea es una técnica que permite un acceso certero y duradero al sistema venoso central, con las ventajas para la monitoría y terapia de los pacientes en estado crítico. Aunque en nuestro trabajo la cateterización se hizo en base a catéteres ad hoc, es útil recordar que es posible tam. bien utilizar "teflones", los que son evidentemente más baratos. Nuestra vía de acceso a la cava superior fue la yugular intema, pero es atjnente recordar que se puede acceder a la cava superior, también, por la yugular externa y por la basílica mediante técnicas y catéteres adecuados.

Como cualquier técnica invasiva, la cateterización percutánea no está exenta de riesgos. La complicación más frecuente citada en la literatura es la punción de la arteria carótida que puede producir, por el hematoma, sindrome de Claude Bernard-Horner ${ }^{4}$. Otras complicaciones citadas en la literatura son el tamponamiento cardíaco ${ }^{6}$ y el hidrotórax ${ }^{7}$, hechos no encontrados en nuestra serie. Sin embargo, si se produjeron en nuestra serie dos neumotórax y en un paciente una bradicardia transitoria ${ }^{8,9}$. El neumotórax se presentó en dos casos que estaban sometidos a ventilación a presión positjva $\mathrm{y}$ ambos requirieron uso de sonda pleural. El cuadro de bradicardia fue pasajero y desapareció al variar la posición del catéter.

Creemos que la cateterización de una vena central por yía percutánea es una técnica que debe ser manejada por pedjatras que tienen a su cargo pacientes críticos; la técnica no es difícil; sin embargo, puede ser riesgosa sin un entrenamiento práctico previo. Es un procedimiento útil para vigilar pacientes, obtención de exámenes, aporte de fluidos como: sueros de diferentes tipos, sangre y derivados, albúmina; o para la administración de alimentación parenteral.

\section{RESUMEN}

Se presenta la experiencia desarrollada en la UCI del Hospital Luis Calvo Mackenna con el uso de catéteres centrales colocados por vía percutánea, utilizando la vena yugular intema. La via de abordaje más utilizada fue la yugular derecha. Se cateterizaron 47 niños en un lapso de 15 meses. Las edades de los pacientes fluctuaron entre 2,5 meses y 14 años y el peso entre 2,5 y $37 \mathrm{~kg}$. La permanencia de los catéteres fue de 4,2 dias, en promedio, con un rango de 1 día a 17 dias. Las complicaciones encontradas fueron: punción arteria carótida en 4 oportunidades, neumotórax en 2 oportunidades $y$ bradicardia transitoria en un paciente. 


\section{REFERENCIAS}

1. Vaughn R.W., Weygandt G.R.: Reliable percutaneous central venous pressure measurement. Anesthesia and Analgesia. Curr Res 1973; 52: 709-716.

2. Shaw J.C.L.: Paraenteral nutrition in the management of sick low birth wejght infants. Pediatr Clin North Am 1973; 20: 333-358.

3. Hermosura B., Vanags L., Dickey M.W.: Measurement of pressure during intravenous therapy. JAMA 1966;195: 321.

4. Prince S.R., Sullivon R.L., Hackel A.: Percutaneous catheterization of internal jugular vein in infants and children. Anesthesiology 1976; 44: 170-174.

5. Michel L.: Infection due to Intravascular Cathe- terization. En Update in Intensive Care and Emergency Medicine. Ed. Springer-Verlag Berlin Heidelbere 1985; 178: 133-136.

6. Greenall M.J., Blewitt R.W., McMahon M.J.: Cardiac tamponade and central venous catheters. $\mathbf{B r}$ Med J 1975; 2: 595-597.

7. Carvell S.E., Pearce D.J.: Bilateral hydrothorax following internal jugular catheterization. Brit 3 Surg 1976; 63: 381-383.

8. Amold S., Feathers R.S., Gibbs E.: Bilateral pneumothoraces and subcutaneous emphysema: a complication of internal jugular venepuncture. Br Med J 1973; $1: 211-212$.

9. Cook T.L., Dueker C.W.: Tension pneumothorax following internal jugular cannulation and genera] anesthesia. Anesthesiology $1976 ; 45 ; 554-555$. 